International Journal of Engineering \&Technology, $7(3.12)(2018) 165-167$
International Journal of Engineering \& Technology
WPC
Website: www.sciencepubco.com/index.php/IJET
Research paper

\title{
Literature Review on High Definition Image Error Concealment
}

\author{
Ghouse Ahamed $Z^{1}$, Anuj Jain ${ }^{2}$ \\ ${ }^{1}$ Research Scholar, ${ }^{2}$ Research Guide \\ ${ }^{1,2}$ Dept of Electronics and Communication, Bhagwanth University
}

\begin{abstract}
This paper is give us a overview of Error control method used in image or video transmission. Data in transmission is lost due to link failure or due to congestion and loss in packets, so the aim of this method is to protect data from these errors. Error detection coding and Error correction coding are two types of error control mechanism. Some of the error control mechanisms are Retransmission, Forward error correction, error concealment and error resilience. We are discussing a summary of three methods and Error Concealment in details.
\end{abstract}

Keywords: Retransmission, Error Concealment, Error Resilience, Forward Error Correction (FEC).

\section{Introduction}

Video or image is first compressed and then divided into packets of variable or fixed length. Packets or data bits may be lost or corrupted, due to either link failure or traffic congestion.

Reconstructed video may become displeasing to human due to a destructive effect caused by Packet loss. The error control mechanisms have been projected in order to increase the video quality in existence of packet loss. The packet loss is unavoidable in the Internet [2-4]. The video communication system designs with error control and congestion control [4] to minimize the packet loss in the Internet.

Depending on the particular network under consideration a number of different types of losses may occur. For example, congestion may cause an entire packet to be discarded (lost), where as wired packet networks such as the Internet are afflicted by packet loss.

In order to correct errors that occur during transmission redundancy is added to information in all error correcting mechanisms [6].

The error control coding is classified into Error detection coding and Error correction coding. In Error detection coding error is identified based on the information received. Since the packet loss causes degradation in visual quality error control mechanism can be used to rectify this problem using Error correction coding. Some of the error control mechanism is forward error correction (FEC), retransmission, and error reseilment and error concealment. Error correcting codes, such as parity, LDPC, Hamming codes [7] and Reed-Solomon [6] used by FEC to correct the error. Parity coding and Reed-Solomon coding are often recommended in (IETF) and Real-Time Transport Protocol (RTP) [8].

In FEC redundant data are added to the compressed packet and transmitted, original image can be reconstructed even if packets are lost. Retransmission mechanisms based on the receiver notifies the sender which packets were received or lost and the sender have

to resend lost packets. ARQ is example of retransmission.
Error Concealments receiver based error control it recover the lost information without the help of the additional information from encoder by using interpolation and estimation.

Error reseilment schemes try to limits the scope of damage created by the packet loss on the compression layer or to prevent propagation of error by adding additional information at the source coding.

This paper is organized as follows in Section 2, it explains types of error Concealment. Section 3, next generation approach .Section 5 conclusion.

\section{Types of Error Concealment}

Based on element used in transmission system which act as a major part in implementation. Error Concealment technique can be divided into three types, namely Forward Error Concealment, Post processing Error Concealment and Interactive Error Concealment. Forward error concealment is done by encoder, Post processing error concealment is done by decoder, and Interactive error concealment is collective done by encoder and decoder.

Forward Error Concealment: Technique can be implemented using techniques all adds some redundancy at the coding stage in order to simplify error recovery process at the decoder. The difference between one techniques to another is how and where redundancy is added .Some examples are Robust Entropy Coding, Channel Coding and Layered coding with transport prioritization In Layered Coding with Transport Prioritization Technique video or image is divided into two layers base layer and Enhancement layer .Base layer is transmitted with maximum priority so that it can produce the video of acceptable quality. Enhancement layer is transmitted with lower priority it contains the information which helps in enhancing the video signal quality.

In Multiple Description Coding technique number of copies of bit streams is produced and each bit stream description is transmitted through different channel.

Occurrence of error in one channel is different from another, so the probability of occurrence of error in all channels is low.

Post processing Error Concealment: This technique is applied at 
the decoder .Received video signal should have a smooth variation in time and space. If there are some irregularities the neighborhood of the affected area can be used to rectify the transmission error. The distortion in high frequencies is tolerated by human visual system this characteristic is also used in this technique. Some examples of this technique are Motion compensated temporal prediction, frequency and spatial domain interpolation, Maximally Smooth recovery, Recovery of Motion vectors and coding modes, Projection onto convex set.

Motion-Compensated Temporal Prediction This technique uses the temporal redundancy of the video sequence in case if error occurs the affected area is replaced by the previous frame. This technique give good results if there are slow movements in video sequence .Motion compensation is better than block compensation.

Maximally Smooth Recovery This technique uses both the temporal and spatial redundancy in order to reduce the difference between pixel values of affected block and temporal and/or spatial neighboring block.

It performs three kinds of interpretations temporal, spatial, frequency (with in block) using received coefficients of the predicted block (obtained from previous blocks) and adjacent blocks of the actual frame.

If the weight of the spatial difference measure is zero, then the technique performs the motion compensated temporal prediction and if the weight of the temporal difference measure is zero, it performs a spatial interpolation with the blocks received in the actual frame, to recreate the affected block.

Interactive Error Concealment These techniques use interaction between coder and decoders. There exist a feedback between coder and decoder. The coder uses information from the decoder to adopt transport mechanism or source coding. Because of this the updated exit at both the end producing better results compare to other methods. Some examples are Adoptive transport for Error concealment, selective encoding for error concealment, Retransmission without waiting and multi copy retransmission.

Selective Encoding for Error Concealment This technique establishes that the decoder should give feedback to the encoder about the spatial location of the affected area. Having this information, the encoder can treat these areas differently to try to eliminate or, at least, reduce the error propagation effects (due to prediction coding) over the video stream at the decoder.

The simplest implementation would be sending the next frame without prediction coding (intramode), whenever an error is detected. From the decoder point of view, whenever it discovers an error, it sends the location information of the affected area to the encoder performs post processing error concealment techniques in that area and continues with normal decoding.

From the encoder point of view, after it has received the feedback from the decoder it chooses from two methodologies. In the first one, the present affected picture area is determined from the frame the decoder indicated up until the actual frame the encoder is working on. This area is not used for prediction coding, at present time. In the second one, the encoder performs the same post processing techniques done by the decoder and reexecutes a local decoding from the concealed block up until the present frame to be encoded. The new decoded frame is used for the present prediction coding.

Retransmission without Waiting, the traditional retransmission system implies that the decoder has to wait for the retransmitted data to proceed. This introduces a delay that can be critical and that, if it surpasses a certain threshold, may create freezes in the decoded video sequence.

The decoder can take two options after having received the retransmission: to decode faster until the decoded frame and its presentation time correspond once again (only a few frames will be shown out of their right presentation time and will result in a quick visual fast forward at the decoded video signal) or to continue to decode at normal speed (a fixed delay is introduced at the decoding process corresponding to the time that took to perform the retransmission).

Either way, a delay appears at the decoding level. This particular technique allows the elimination of any delay associated to the retransmission. In this technique, after a retransmission request the decoder doesn't wait and applies post processing techniques to the damaged area and continues the normal decoding (and displaying) while, in parallel, performs and records a trace of the error affected area (it spreads throughout the image due to the prediction coding).

Once the retransmission arrives, the decoder uses this information and the trace made before to correct the present affected area. From that point, it can continue as if no errors have ever occurred. With this scheme, this technique achieves lossless recovery except during the time the information got erroneous and the retransmitted data arrives to the decoder.

\section{Next Generation Approach}

The coding algorithm was initially established in a pixel oriented scheme. Later, it was introduced a new scheme of codification (an object based orientated one) where the superposition of various objects is what generates an image

.Object based generation of an image. The error concealment techniques had to adapt themselves to this new coding scheme and had to be oriented to an object based configuration also.

The idea is to use statistical models (2-D or 3-D) to shape the objects that appear in the image and use them to replenish the lost data. Examples of this kind of techniques is Model-Based Error Concealment

Model-Based Error Concealment for Wireless Video This technique is based in two steps: first, projection onto the model to obtain the reconstruction for the object; second, replace the lost data using the reconstruction created in the previous step. Like this, a model has to be established and for that, models that involve linear combinations of a set of basis vectors to reconstruct the object are used.

Hence, the set of reconstructions obtained, when using these models, is convex. Furthermore, the set of objects or regions of interest is also convex. Like this, both operations are projections onto convex sets, and the process can be iterated to give better results. This technique can be used with any object-based video standard such as the MPEG-4 standard.

\section{Conclusion}

In this paper, we have described the error control mechanisms for video application, which can reduce the packet loss in order to provide good video quality. Existing error control mechanisms can be classified into four types, namely, forward error correction (FEC), retransmission, error resilience, and error concealment. The first two are in channel coding and the latter two are in source coding. Then error concealment is explained in detail. Forward error concealment techniques trade off coding efficiency for error resilience and usually need more complex encoders.

Post processing error concealment techniques do not involve extra redundancy and can be applied at any video system. Interactive error concealment techniques require feedback information (that may be not available,e.g., video broadcast systems) to establish the cooperation between coder and decoder. Second generation error concealment techniques establish statistical models for the various objects that compose the image and use them, whenever an error occurs, to replenish the affected area.

\section{References}

[1] D. Wu., Y. T. Hou., and Y.-Q. Zhang., "Transporting Real-Time Video over the Internet: Challenges and Approaches," Proceedings of the IEEE, vol. 88, pp. 1855-1877, Dec2000.

[2] Jafar Ababneh and Omar Almomani." Survey of Error Correction Mechanisms for Video Streaming over the Internet," Internationa Journal of Advanced Computer Science and Applications, Vol.5, No. 3,2014.

[3] Y. Wang, A. R. Reibman, and S. Lin, "Multiple Description Coding for Video Delivery," Proceedings of the IEEE, vol. 93, pp. 57 
$70,2005$.

[4] D. Wu., Y. T. Hou., W. Zhu., Y.-Q. Zhang., and J. M. Peha., "Streaming Video over the Internet: Approaches and Directions," Circuits and Systems for Video Technology, IEEE Transactions on, vol. 11, pp. 282-300, Mar2001.

[5] Robert.H and Morelos-Zaragoza, The Art of Error Correcting Coding. England: John Wiley \& SonsLtd,, 2006.

[5] Chen, T. P.-c. and Chen, T. (2002). Second-generation error concealment for video transport over error prone channels. Image Processing. 2002. Proceedings. 2002 International Conference on, $1: \mathrm{I}-25-\mathrm{I}-28$.

[6] Khansari, M. and Vetterli, M. (1995). Layered transmission of signals over powerconstrained wireless channels. Image Processing, 1995. Proceedings., International Conference on,3:380-383.

[7] Kieu, L. H. and Ngan, K. N. (1994). Cell-loss concealment techniques for layered video codecs in an atm network. Image Processing, IEEE Transactions on,3(5):666-677.

[8] Turaga, D. S. and Chen, T. (2002). Model-based error concealment for wireless video. Circuits and Systems for Video Technology, IEEE Transactions on,12(6):483-495.

[9] Wada, M. (1989). Selective recovery of video packet loss using error concealment. Selected Areas in Communications, IEEE Journal on, 7(5):807-814. 